\title{
DÉCLIN ET RESTAURATION DE LA POPULATION DE TRUITES LACUSTRES (SALMO TRUTTA LACUSTRIS L.) DU LAC DE CONSTANCE
}

\author{
par Ch. RUHLE \\ Service de la Chasse et de la Pêche du Canton de St-Gall \\ Burggraben 26 - $\mathrm{CH}-9001 \mathrm{ST}-\mathrm{GALL}$ (Suisse)
}

\section{RÉSUMÉ}

Le déclin de la population de truites migratrices du lac de Constance a débuté vers 1950 quand un barrage a été construit sur le Rhin, affluent principal de ce lac, coupant ainsi l'accès aux plus importantes frayères. Ceci a suffi de prétexte pour renoncer à une longueur de capture garantissant la reproduction naturelle. Par la suite, la population en train de diminuer a servi de justification pour des déversements importants de truites arc-en-ciel. Un désintérêt croissant pour le maintien de frayères dans les affluents secondaires $a$, finalement, contribué à la baisse des captures de $12.000 \mathrm{~kg}$ à $3.000 \mathrm{~kg}$ entre 1950 et 1980 .

Les mesures réalisées au cours des dernières années pour restaurer la population de truites lacustres ont du succès. II s'agit surtout de :

- la détermination d'une nouvelle longueur de capture ainsi que de périodes de protection respectant la reproduction des truites migrant à longue et à courte distance,

- l'élevage de stocks de géniteurs,

- les déversements forcés et effectués surtout en affluents (au lieu du lac),

- l'interdiction d'immersions de truites arc-en-ciel,

- l'abolition de barrages.

Le succès se manifeste par un nombre croissant de truites observées frayant dans les affluents ainsi que par des captures de plus en plus nombreuses faites par la pêche professionnelle.

\section{DECLINE AND RESTORATION OF THE POPULATION OF MIGRATING LAKE-TROUT (SALMO TRUTTA LACUSTRIS L.) OF LAKE CONSTANCE}

\section{SUMMARY}

The decline of the population of migrating lake-trout of Lake Constance started at the beginning of the $1950 \mathrm{~s}$ when a dam was built up in the most important tributary, the Rhine, preventing the spawners from the access to the most important spawning grounds. On the pretext that a natural reproduction could no longer occur, the former size limit ensuring the ripening of the trouts was abandoned and rainbow trouts were heavily stocked to compensate the decline of the autochthonous trout population. Finally also spawning ground of poorer importance were neglected and the catches dropped from $12,000 \mathrm{~kg}$ to $3,000 \mathrm{~kg}$ within 30 years.

Different measures applied in the last years to restore the declining autochthonous trout population are obviously successful :

- three-year-closure for the trout-selective angling,

- determination of a size limit and of closed seasons considering the reproduction of far and of short-distance migrating trouts,

- rearing of parental stocks,

- stockings done in tributaries instead of lake,

- suppression of rainbow-trout stockings.

- abolition of migrating barriers.

Increasing catches of trouts in the commercial fishery as well as reestablished migrations in some accessible tributaries point to a progressing restoration.

\section{INTRODUCTION}

L'accomplissement du cycle vital de la truite lacustre exige, en général, deux milieux différents: les fleuves et les rivières lui servent de milieu de reproduction tandis que les lacs sont milieu de croissance. Par cela, leurs populations sont soumises à un impact humain plus complexe que si elles vivaient dans un seul milieu.

Les populations de truite lacustre en Suisse ont toutes souffert sous cette accumulation d'influences humaines négatives. Les unes ont disparu et pour d'autres le déclin est ralenti ou freiné 
grâce à des contre-mesures coûteuses. La population de truite lacustre du lac de Constance (fig. 1, Tab. 1) a été soumise en peu de temps à une série de menaces partiellement enchaînées les unes aux autres. Son déclin qui en est ressorti a pu se manifester d'une façon plus évidente que dans d'autres cas et différents appuis ont été lancés avant son extinction.

\section{Lac de Constance localisation des impasses}
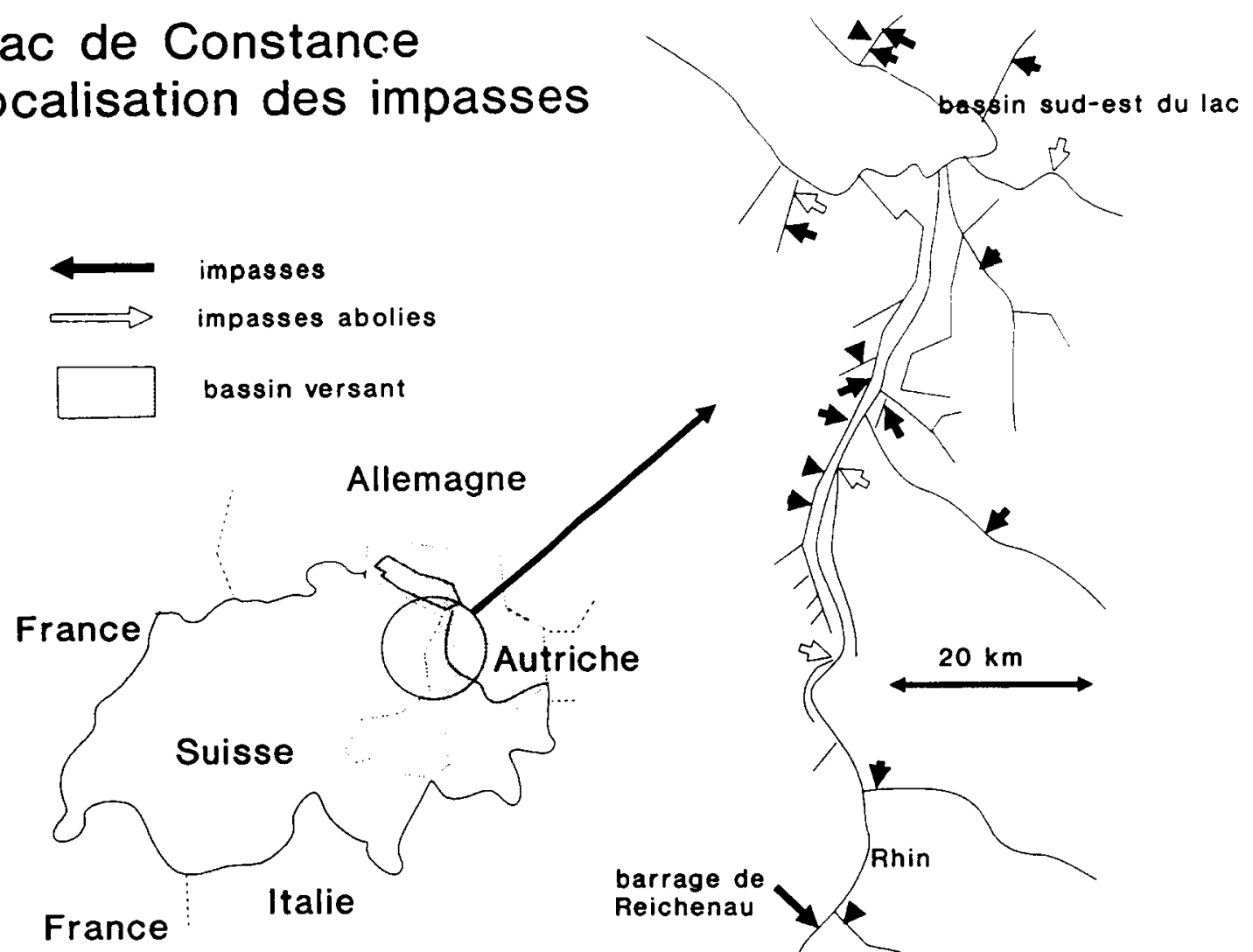

fig. 1

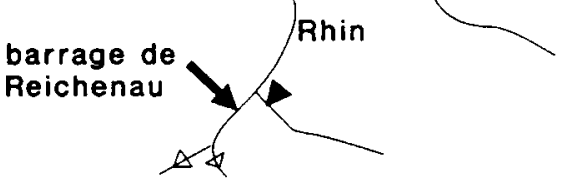

figure 1 : Situation géographique : Suisse et lac de Constance avec bassin versant et localisation des impasses.

figure 1 : Study area : geographic location of Lake Constance and tributaries and of migrating barriers.

\begin{tabular}{|c|c|c|}
\hline Table | & : Lake Constance characteri & stics \\
\hline & $\begin{array}{l}\text { Surface } \\
\text { Volume } \\
\text { Profondeur maximale } \\
\text { Profondeur moyenne } \\
\text { Bassin versant } \\
\text { Temps de renouvellement } \\
\text { Etat trophique }\end{array}$ & $\begin{array}{lc}: & 539 \mathrm{~km}^{2} \\
: & 49,4 \mathrm{~km}^{3} \\
: & 252 \mathrm{~m} \\
: & 92 \mathrm{~m}^{2} \\
: & 10.900 \mathrm{~km}^{2} \\
: & 4,6 \text { années } \\
: & \text { mésotrophe }\end{array}$ \\
\hline
\end{tabular}

\section{OBJETS D'ÉTUDES}

Le lac de Constance est situé entre la Suisse au sud-ouest, l'Allemagne au nord-est et l'Autriche au sud-est. Ses caractéristiques principales sont résumées dans le Tableau 1.

La pêche effectuée par environ 170 pêcheurs professionnels et environ 3.500 pêcheurs sportifs (sans la pêche côtière) est axée sur les corégones et les perches. La capture annuelle moyenne des derniers dix ans était de $1.223,5$ tonnes ou de $25,7 \mathrm{~kg} / \mathrm{ha}$. Les deux espèces mentionnées représentent $30 \%$ et $37 \%$. Le reste se compose de brochets, d'anguilles, d'ombles chevaliers, 
de truites lacustres et arc-en-ciel ainsi que de différentes espèces de Cyprinidés et d'autres poissons (en très petite quantité : sandres, silures). Pour la période des 10 années passées la truite lacustre représente environ $0,2 \%$ des captures totales $(\mathrm{kg})$. Entre 1920 et 1950 ce pourcentage était de 2 à $3 \%$.

Les données de croissance des truites lacustres sont présentées dans la figure 2. Les poissons considérés ont été capturés entre 1975 et 1982 et proviennent probablement de repeuplements effectués dans le lac même (frayères inaccessibles).

La maturité est atteinte, en général, à l'âge $4^{+}$ou $5^{+}$à une longueur de $50 \mathrm{~cm}$ ou plus (RUHLÉ, 1983: RUHLÉ et al., 1984). La maturation saisonnière diffère en fonction des distances à migrer entre le lac et les frayères : elle débute plus tôt pour les individus migrant à longues distances.

\section{Truites lacustres du lac de Constance Croissance en longueur et en poids}

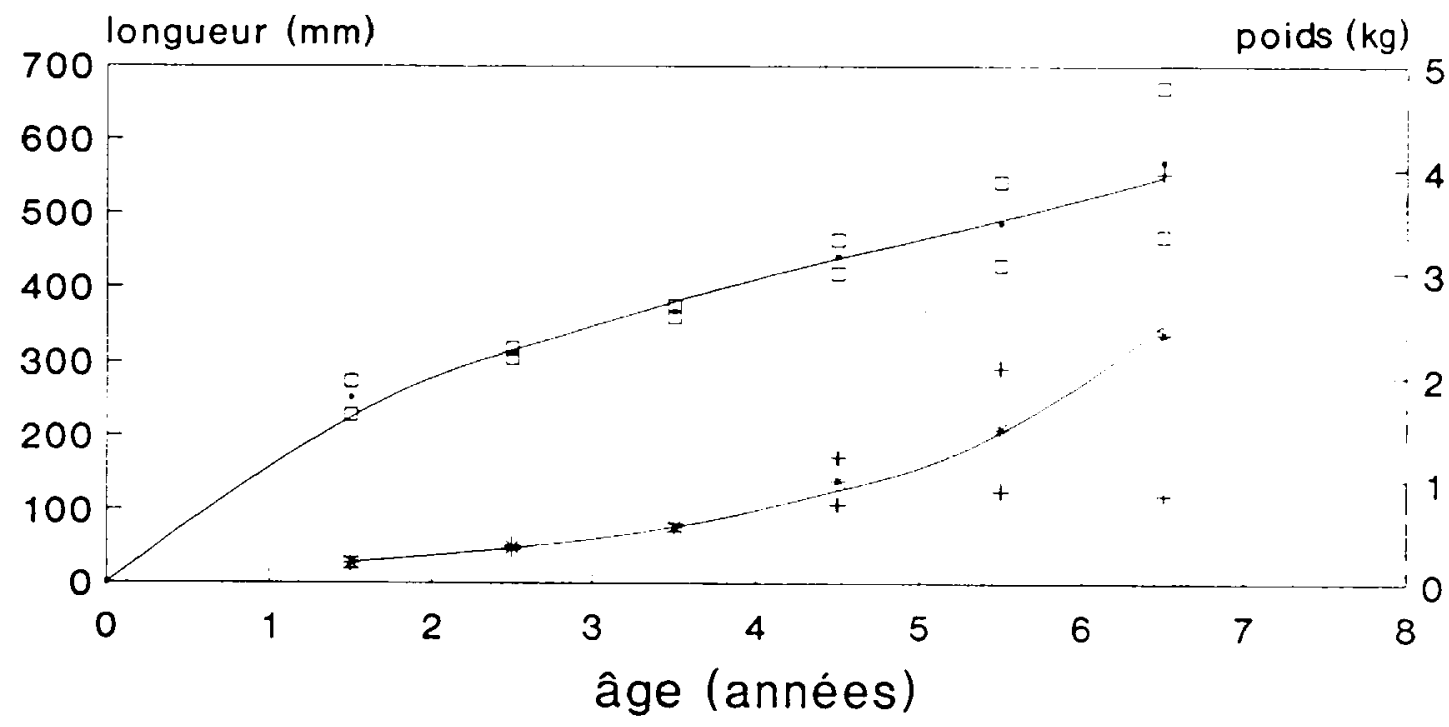

\footnotetext{
- longueur moyenne

$\because \quad$ LC $(95 \%)$ longueur

* poids moyen

- LC $(95 \%)$ poids
}

figure 2 : Croissance en longueur et en poids des truites lacustres.

figure 2 : Growth pattern of L. Constance lake-trout (length and weight).

\section{DÉCLIN DE LA POPULATION DE TRUITES LACUSTRES}

\section{1. Évolution des captures}

L'évolution des captures de la pêche professionnelle dans le lac de Constance est présentée dans la figure 3. L'époque comprise entre 1915 et 1985 peut être divisée en trois périodes

- 1915-1945 : captures stables d'environ 11.000 à $12.000 \mathrm{~kg}$

- 1945-1955 : augmentation des captures

- 1955-1985 : baisse des captures.

La première période est caractérisée par des conditions de pêche constantes et une reproduction naturelle assurée, la deuxième reflète la situation déterminée par l'eutrophisation progressante du lac et par une pêche professionnelle intensifiée (introduction de filets monofilaments) et pendant la troisième, les impacts négatifs se manifestent dans un déclin prononcé. 


\section{Captures de truites lacustres par la pêche professionnelle du lac de Constance}

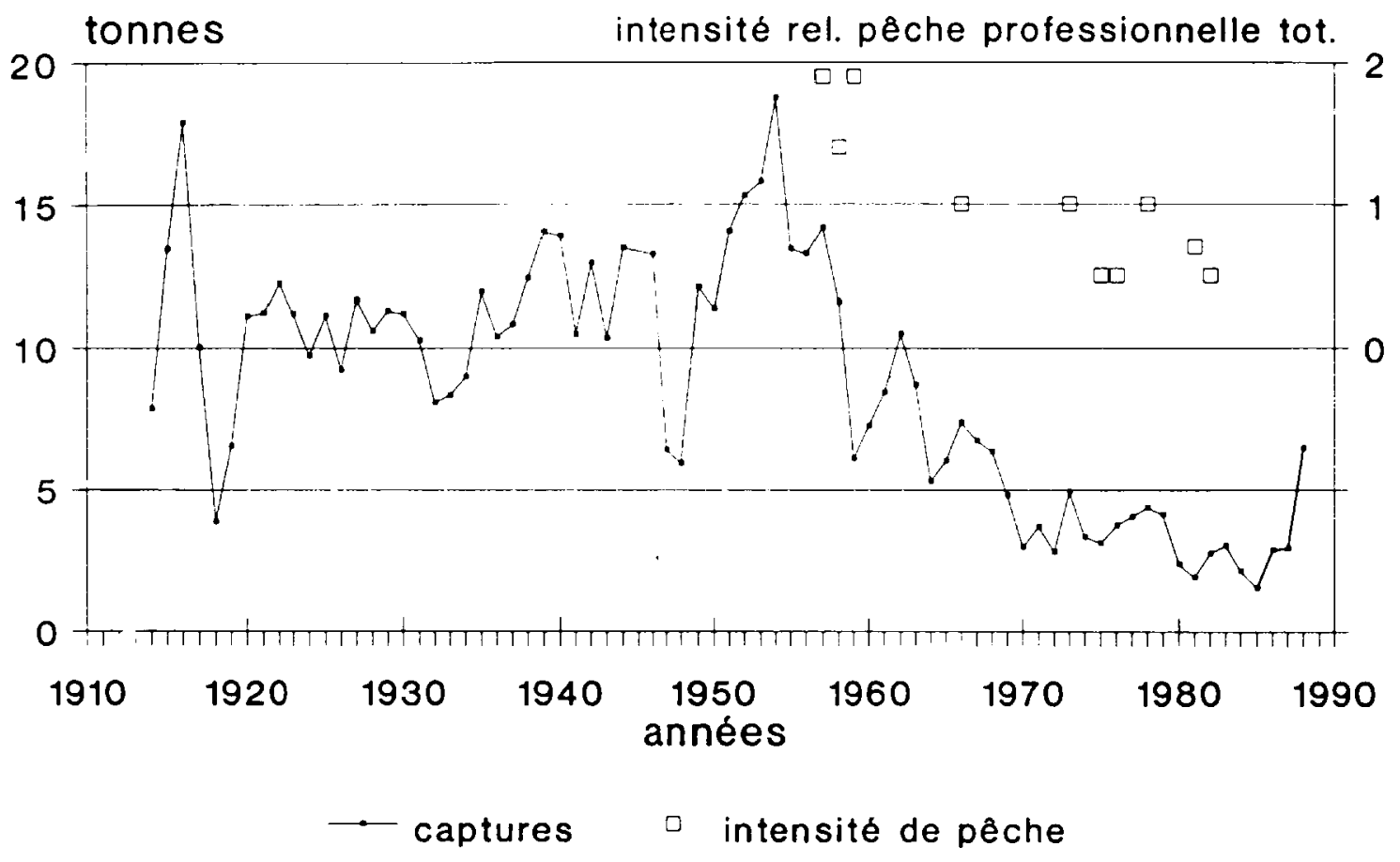
figure 3 : Évolution des captures de truites lacustres réalisées par la pêche professionnelle dans le lac de Constance entre 1915 et 1987 et intensité de pêche relative (d'après HARTMANN, comm. orale).

figure 3 : Yields (tons) of lake trouts in the commercial fishery 1915-1987, and relative fishery effort.

\subsection{Impact primordial et engrenage de menaces}

Au cours de la première moitié de ce siècle, différents petits affluents du lac de Constance ou de ses tributaires ont perdu leur fonction d'aires de reproduction soit à cause de la construction de barrage empêchant la migration soit à cause de la pollution croissante. Ces tronçonnages n'ont pas influencés essentiellement la population des truites lacustres (fig. 3).

La construction du barrage de Reichenau, entre 1955 et 1957, a, d'autre part, exercé un rôle dominant pour le destin des truites lacustres dans deux sens:

- le barrage a coupé l'accès aux frayères importantes dans un système d'affluents d'environ $90 \mathrm{~km}$ de longueur,

- sa construction a provoqué des modifications de la gestion de la pêche des truites du lac de Constance :

- Sous prétexte que le barrage ait anéanti la reproduction naturelle, la longueur de capture respectant la biologie de reproduction a été abandonnée. Cette longueur légale de capture, en vigueur en particulier pour les pêches sélectives des pêcheurs sportifs, a été, par la suite, adaptée aux longueurs des poissons capturés dans les filets flottants de la pêche professionnelle (1955: $40 \mathrm{~cm}, 1978: 35 \mathrm{~cm})$.

- La population des truites lacustres soumise à une pêche intensifiée et dépourvue des frayères principales n'a plus réussi à produire des géniteurs en nombre suffisant pour se maintenir (fig. 4).

- La disparition successive des géniteurs des frayères encore accessibles a fait tolérer la construction d'autres obstacles. 


\section{Captures de géniteurs dans la pêche de reproduction (barrage de Reichenau)}

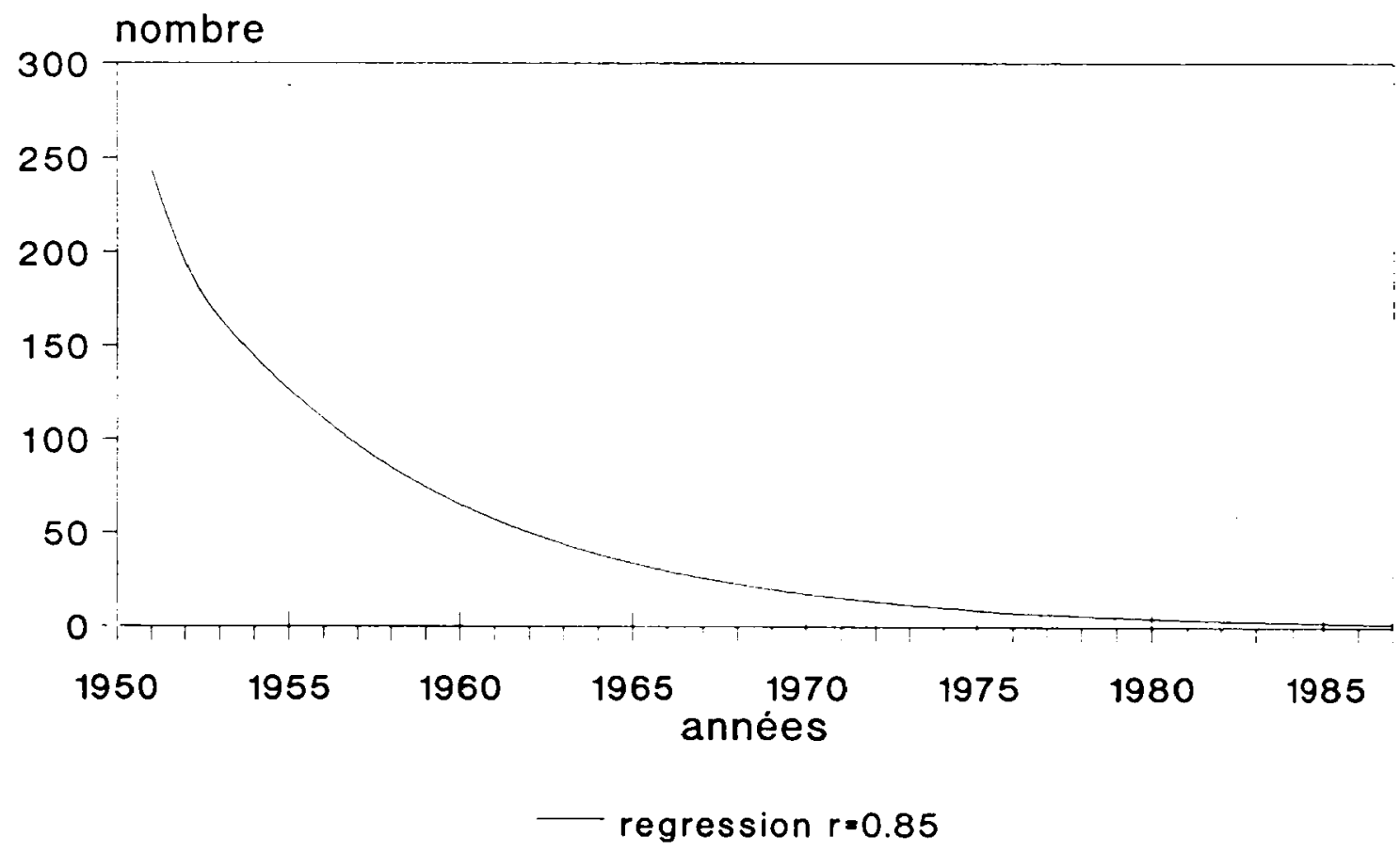

figure 4 : Évolution des captures de géniteurs (pêche de reproduction) en aval du barrage de Reichenau entre 1952 et 1986.

figure 4 : Spawning fishery downstream from the dam at Reichenau : evolution of the catches 1952-1986.

- Le déclin de la population de truites lacustres a favorisé les repeuplements de truites arc-enciel. Celles-ci concourent les truites indigènes pour les frayères encore en fonction (KINDLE, 1983; HAYES, 1987 ; PETER, 1988).

Vers 1980 des projets de construction pour 14 barrages dans le Rhin ont été présentés au public. Au moment où il fallait réclamer leur adaptation aux exigences des poissons migrateurs, en particulier des truites lacustres, ce poisson avait pour ainsi dire presque complètement disparu à cause de cet enchainement fatal d'impacts menaçants.

\section{CONTRE-MESURES}

L'analyse des causes menant à la disparition presque totale de la truite lacustre du lac de Constance a fait reconnaître la complexité des menaces et la nécessité d'appliquer simultanément des contre-mesures sur différents plans.

\subsection{Longueur légale de capture et saisons de protection}

L'application de nouvelles mesures assurant la reproduction naturelle (longueur légale, saisons de protections) a été basée sur les résultats d'expériences faites au début des années 1980 . Aujourd'hui la pêche se fait sous les conditions suivantes :

- longueur légale (depuis 1983) : $50 \mathrm{~cm}$,

- interdiction de capturer des truites de plus de $45 \mathrm{~cm}$ entre le $1^{\text {er }}$ août et le 31 janvier dans les tributaires importants.

- Saisons de protection dans le lac (depuis 1987) : 15 juillet au 15 septembre (protection des migrateurs à longue distance) ainsi que $1^{{ }^{2 r}}$ novembre au 10 janvier (protection des migrateurs à courte distance). 


\subsection{Amélioration de l'accessibilité aux frayères}

Des passes à poissons ont été construites dans différents tributaires (fig. 1).

\subsection{Elevage de stocks géniteurs et repeuplements des affluents}

La fraie prélevée sur les rares géniteurs observés en migration ou sur les frayères a été utilisée pour l'élevage de stocks de géniteurs. Aujourd'hui, ceux-ci comprennent 5.000 truites d'âge $0^{+}$, 2.200 truites $1^{+}, 300$ truites $2^{+}, 1.100$ truites $3^{+}$et 930 truites $5^{+}$de différentes souches, c'est-à-dire de différents affluents.

Pour les repeuplements, faits en majeure partie dans les affluents pour favoriser un homing éventuel, des alevins provenant de ces stocks et de géniteurs capturés pendant les pêches de reproduction sont utilisés. La mise à disposition d'un grand nombre d'alevins de truites lacustres a permis l'interdiction de tout repeuplement de truites arc-en-ciel.

\section{EFFICACITE DES CONTRE-MESURES ET FUTURS PROBLÈMES}

Le succès des contre-mesures se manifeste, pour l'instant, sous deux formes:

- les captures de truites lacustres réalisées par la pêche professionnelle ont augmenté de $1.500 \mathrm{~kg}$ minimum absolu) en 1985 à presque $6.500 \mathrm{~kg}$ en 1988 (fig. 3),

- dans différents tributaires rendus accessibles par des passes à poissons (fig. 1), un nombre croissant de géniteurs peut être capturé dans les pêches à reproduction. La fraie obtenue par ces captures permet une augmentation des repeuplements.

Malgré ces succès, de graves problèmes restent à être résolus :

- L'amélioration de l'accessibilité des tributaires qui a été jugée un des moyens primordiaux pour la restauration de la population des truites lacustres doit, aujourd'hui, être soumise à une nouvelle consideration : dans certains tributaires secondaires qui ne contenaient auparavant que des truites de rivière, la population piscicole se compose, peu d'années après l'installation des passes à poissons, d'une fraction croissante de truites arc-en-ciel provenant du lac et des tributaires principaux. Elles se reproduisent avec succès et le décalage des saisons de reproduction des truites de rivière et des truites arc-en-ciel contient le risque que des superimpositions des frayères au désavantage des truites indigènes (HAYES, 1987; PETER, 1988) se fassent. Dans la restauration de la population des truites lacustres du lac de Constance, l'orientation initiale et exclusive envers des mesures favorisant l'espèce menacée doit dorénavant être remplacée ou complétée par des mesures réduisant la concurrence interspécifique entre truites fario et arc-en-ciel, surtout dans les aires de reproduction (interdiction de repeuplements de truites arc-en-ciel, mise hors vigueur de la taille légale de capture pour celles-ci).

- La pêche professionnelle orientée vers la capture de corégones et de perches est hors mesure d'éviter les prises de truites lacustres qui n'ont pas la taille légale. Les pêcheurs sportifs demandent de pouvoir participer à ces captures et exigent une réduction de la taille légale. Ces demandes nous ramèreraient au début des problèmes discutés ici.

\section{BIBLIOGRAPHIE}

HAYES J.W., 1987. Competition for spawning space between Brown (Salmo trutta) and Rainbow Trout (S. gairdneri) in a lake inlet tributary, New Zealand. Can. J. Fish. Aquat. Sci., 44 (1) : 40-47.

KINDLE T., 1983. Natürliche Vermehrung der Regenbogenforelle im Liechtensteiner Binnenkanal. Oesterreichs Fischerei, $36: 7-8$.

PETER A., 1988. Zur Konkurrenzierung zwischen Bach-und Regenbogenforelle im Kanalsystem des st. gallischen Rheintals. 1. Bericht polycopiert, unveröffentlicht.

RUHLE Ch., 1983. Wachstumsverhältnisse und Reifeentwicklung bei der Seeforelle (Salmo trutta lacustris L.) des Bodensees. Oesterreichs Fischerei, 36 : 196-201.

RUHLÉ Ch., DEUFEL J., KEIZ G., KINDLE T., KLEIN M., LOFFLER H., et WAGNER B., 1984. Die Bodensee-Seeforelle Probleme und Problemlösungen. Oesterreichs Fischerei, 37 : 272-307. 